\title{
Spinal Neurostimulation for Pain Control: A Review
}

\author{
Tae-Kyu Lee, Kyung-Souk Cho ${ }^{\varpi}$ \\ Department of Neurosurgery, Uijeongbu St. Mary's Hospital, The Catholic University of Korea College of Medicine, Seoul, Korea
}

\begin{abstract}
Management of pain by neurostimulation began shortly after Melzack and Wall proposed the gate control theory in 1965. Spinal neuromodulation for pain relief is most frequently delivered through spinal cord of cervical and thoracolumbar region. The stimulation of these regions by spinal cord stimulation (SCS) has proven its effectiveness for treating a number of neuropathic and nociceptive pain states that are not responsive nor amenable to medication and other known therapies. SCS is somewhat different intracranial neurostimulation for the treatment of medically intractable pain. However, the increasing use of SCS for the treatment of chronic pain, especially for pain not responsive to other neuromodulation techniques, reflects the efficacy and relative safety of these epidural procedures. In 1971 , Shimoji and colleagues first reported the analgesic properties of epidural spinal cord stimulation. Since then SCS has undergone numerous technical and clinical developments. SCS represents one of the earliest uses of neuromodulation to treat chronic pain that is refractory to medical therapy. Currently, two kinds of SCS are commonly used to control pain: percutaneous lead technique and surgical lead after laminectomy. SCS has shown particular promise in the treatment of a number of nociceptive and neuropathic pain states, including post spinal surgery syndrome (PSSS), complex regional pain syndrome (CRPS), post-herpetic neuralgia (PHN), ischemic pain, peripheral vascular disease (PVD), angina, visceral pain and pelvic pain. This article will review the scientific rationale, indications, surgical techniques, and outcomes of SCS for the treatment of chronic pain.
\end{abstract}

Key Words: Spinal cord stimulation; Chronic pain; Neuropathic pain.

\Corresponding author: Kyung-Souk Cho, Department of Neurosurgery, Uijeongbu St Mary's Hospital, The Catholic University of Korea College of Medicine, 271 Cheonbo-ro, Uijeongbu 11765, Korea. Tel: 82-31-820-3638, Fax: 82-31-820-2041, E-mail: magpie67@catholic.ac.kr

\section{INTRODUCTION}

Neuromodulation is the technology impacting the neural network and interface. The INS (the International Neuromodulation Society) defines neuromodulation as a field of science, medicine, and bioengineering that encompasses implantable and non-implantable technologies, electrical or chemical, for the purpose of quality of life and functioning of humans. At the present time, neuromodulation implantable devices are either neural stimulators or micro infusion pumps. These devices are being utilized for the management of chronic pain, movement disorders, psychiatric disorders, epilepsy, movement disorders, disorders of pacing, spasticity, and others. As a neuromodulation for chronic pain, SCS can be an effective alternative or adjunct treatment to other known therapies that have failed to manage pain on their own. SCS delivers small electrical signals by a lead implanted in the epidural space. Pain signals are inhibited before they reach the brain. Instead of pain, patients may feel pain relief. It has generally been accepted that SCS relieved pain in various patients with chronic and medically intractable pain (Fig. 1). This review describes the introduction of SCS, the surgical technique of SCS, the efficacy, complication in which they occur, and contemporary SCS literature.

\section{SPINAL CORD STIMULATION}

The control of pain by neurostimulation began shortly after Melzack and Wall proposed the "gate control theory" in $1965^{14)}$. SCS came into clinical practice as a direct spin-off from the gate control theory, Shimoji and colleagues first reported the analgesic properties of epidural spinal cord stimulation in $1971^{22}$. This therapy has been used to treat neuropathic pain as well as ischemic pain, and visceral and pelvic pain. The neurophysiologic mechanisms of SCS are not completely understood, however, some research suggests that its effects occur at local and supraspinal levels and also through dorsal horn interneuron and neurochemical mechanisms. Experimental evidence supports a beneficial SCS effect at the dorsal horn level, whereby the hyper- 

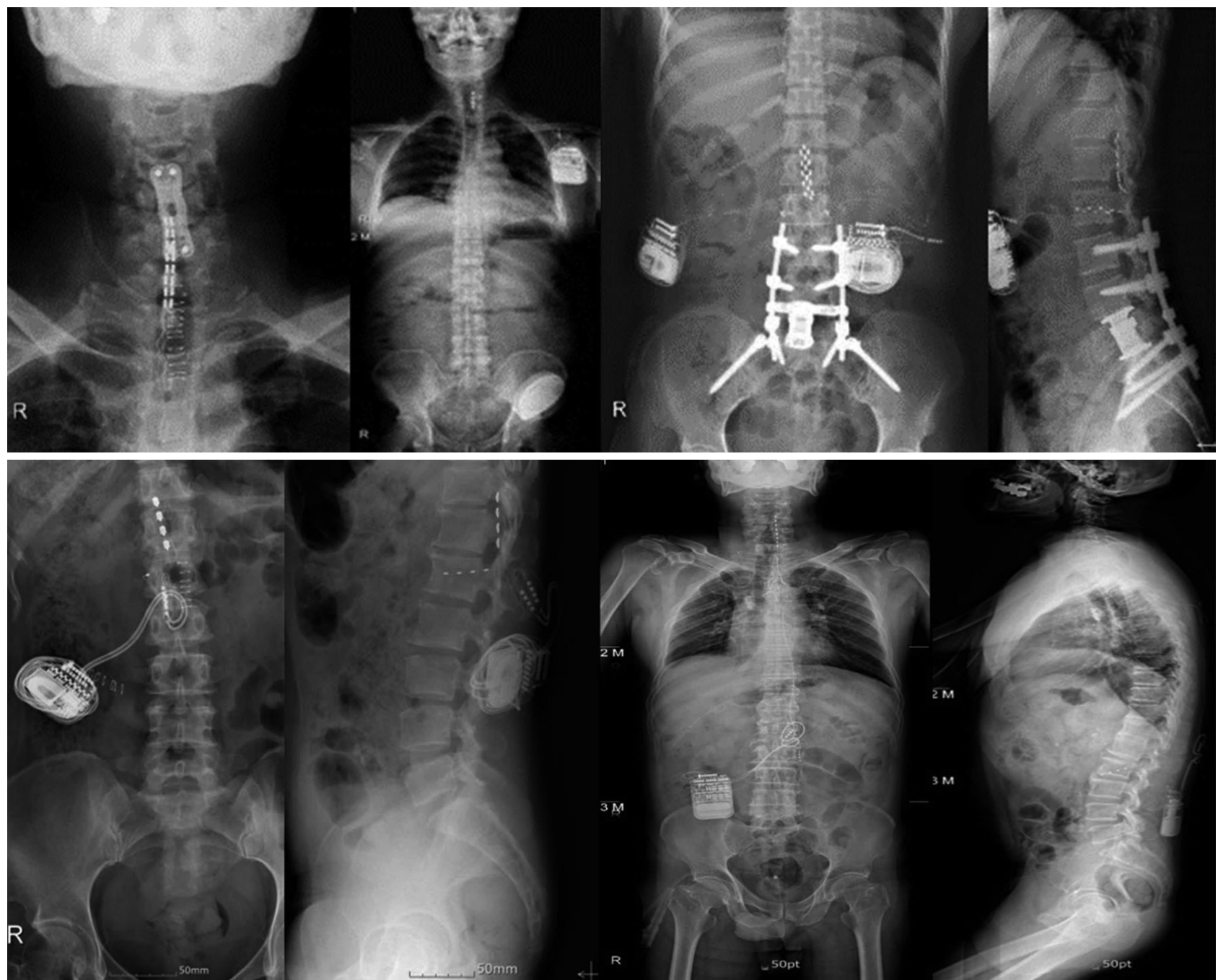

Fig. 1. Plain X-ray shows SCS as an aid in the management of chronic, intractable pain of the trunk and/or limbs-including unilateral or bilateral pain associated with PSSS after cervical spine surgery (left upper), PSSS after lumbar spine surgery (right upper), PVD with diabetes (left lower), and CRPS (right lower).

excitability of wide-dynamic-range neurons is suppressed. Evidence exists for increased levels of gamma-amino butyric acid (GABA) release and serotonin, and perhaps, for reduced levels of some excitatory amino acids, such as glutamate and aspartame ${ }^{5}$.

\section{Patient selection for SCS}

Patient selection is the most important aspect to result in a successful outcome and for increased patient satisfaction. The most relevant issues are patient characteristics, and state of disease causing the pain syndrome.

\section{Patient characteristics}

1. Abuse or abnormal behavior with opioids

2. Presence of psychiatric and psychological disease.

3. Inability to understand risks and benefits of SCS.

4. Presence of bleeding tendency.

5. Presence of infection at the site of implant or systemically. 6. Physician impression that the patient is a poor candidate.

\section{Disease state causing the pain syndrome}

SCS as an aid in the management of chronic, intractable pain of the trunk and/or limbs-including unilateral or bilateral pain associated with the following conditions:

1. Peripheral vascular disease with diabetes

2. Angina and ischemic pain

3. Post spinal surgery syndrome (PSSS) or low back syndrome

4. Complex regional pain syndrome (CRPS type I, II)

5. Diabetic neuropathy

6. Stump pain

7. Phantom limb pain

8. Incomplete spinal cord injury

\section{SURGICAL TECHNIQUE FOR SCS}

\section{Epidural lead placement via percutaneous technique}

The implantation is carried out in the operating room using sterile technique. Minimal or no preoperative sedation is given. The patient is placed in either the lateral or the prone position on an $\mathrm{x}$-ray transmitting table. Two spinous interspaces are se- 
lected for their proximity to the spinal segment innervating the painful area. Tuohy-type needles are advanced through the interspaces into the epidural space, which is identified by the negative pressure method or by loss of resistance. The electrodes are inserted through the needles and advanced in the epidural space so that their tip rests one or more interspaces cephalad to the tip of the respective needles (Fig. 2). The position is checked at intervals by biplane radiograph or fluoroscopy with image intensification. The electrodes are then connected to the stimulating unit and test stimulation is carried out using various frequencies and intensities. The electrodes are navigated under fluoroscopic control so that stimulation produces maximum sensations on the pain site. If the correct reference of paresthesia is achieved, the electrode system is implanted by connecting the electrodes subcutaneously to the receiver which is placed in a subcutaneous pocket in the left flank. At each step during the surgical implantation of the system stimulation is carried out to be certain that the paresthesia has not been altered ${ }^{16)}$.

\section{Epidural placement of surgical lead via laminectomy}

To place electrodes that are implanted via laminectomy, the principles are the same. The entry laminectomy is usually at optimal interspace so that the electrode tip ends at the top of adjacent vertebra or bottom of upper vertebra. The paraspinal muscles are carefully stripped from the spine and lamina at one interspace. A partial laminectomy, large enough to allow passage of the electrode, is performed. The epidural space is cleared and
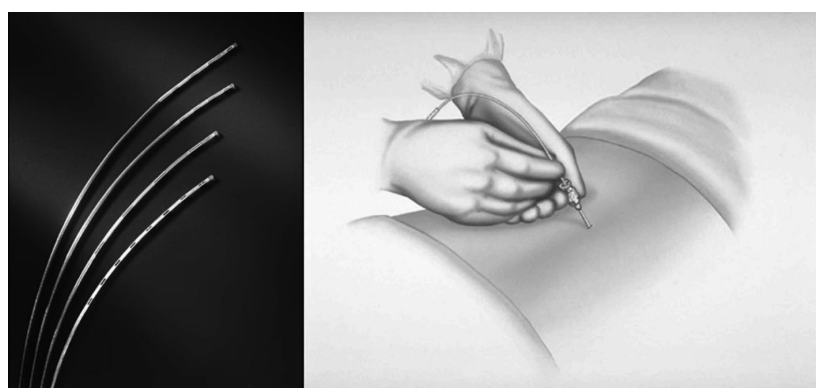

Fig. 2. The percutaneous electrodes (left) are inserted through a Tuohy needle and advanced in the epidural space so that their tip rests one or more interspaces cephalad to the tip of this needle for minimally invasive lead placement (right).
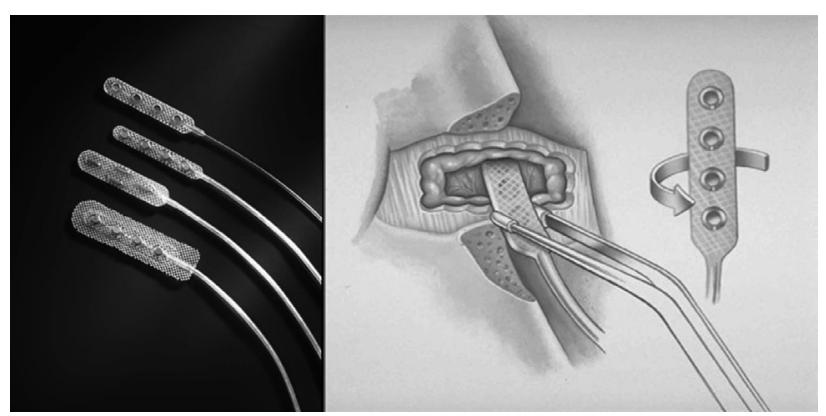

Fig. 3. A partial laminectomy (right), large enough to allow passage of the surgical electrode (left), is performed. The epidural space is cleared and the size of the opening is confirmed using the lead blank. The electrode is then carefully advanced in the epidural space with the electrodes facing the dura. the size of the opening is confirmed using the lead blank. The electrode is then carefully advanced in the epidural space with the electrodes facing the dura (Fig. 3). The position of the lead is then confirmed by test stimulation (Table 1). It is occasionally necessary to enlarge the laminectomy opening to allow greater manipulation of the electrode position; however, this makes the procedure more uncomfortable for the patient. If enough dura is exposed, the electrode can be sutured to the dura. One of the reported advantages of the surgical electrode is reduced migration, but this is achieved at the expense of a more difficult procedure. The surgical electrode is not migration free ${ }^{16}$.

\section{Preoperative preparation before implantation of internal pulse generator (IPG)}

Almost all surgeons now employ some form of trial stimulation prior to implanting SCS system. Especially, a percutaneously placed electrode meets the needs for trial stimulation well, since it is relatively noninvasive. Trial stimulation allows the patient to experience the paresthesia induced by stimulation

Table 1. Sensory mapping in spinal cord stimulation

\begin{tabular}{lll}
\hline \multicolumn{2}{c}{ Pain location (dermatome) } & Highest probability of electrode \\
\hline Anterior shoulder & C4 & C3 (C3-5) \\
External arm & C5 & C4 (C2-T3) \\
Radial forearm & C6 6 C5 (C2-T3) \\
Median hand & C6-7 & C6 (C2-T2) \\
Ulnar hand & C8 & C7 (C2-T2) \\
Ulnar forearm & T1 & C7 (C4-T3) \\
Internal arm & T2 & T1 (C5-T3) \\
Chest & T2-T6 & T2 (T1-T7) \\
Low back & T9-L1 & T9 (T8-T11) \\
Abdomen & T9-L1 & T8 (T6-T11) \\
Anterior thigh & L2-3 & T11 (T11-T12) \\
Anterior leg & L4-5 & T12 (T12-L1) \\
Foot only & L5-S1 & L1 (T11-L1) \\
Posterior leg & S1-2 & L1 (T11-L1) \\
Posterior thigh & S1-2 & L1 (T11-L1) \\
Buttock and leg & T9-10 & T9-10 (T11-L1) \\
\hline
\end{tabular}

Table 2. Efficacy of SCS for various pain syndrome

\begin{tabular}{ll}
\hline Efficacy & Pain syndrome \\
\hline Very effective & PVD (w/diabetic) \\
& Angina/Ischemic pain \\
Effective & PSSS (Lower limb) \\
& CRPS (Type 1, 1l) \\
& Diabetic Neuropathy \\
& Stump pain \\
Mild & Phantom limb pain \\
& Incomplete spinal cord injury \\
& PSSS (lower back) \\
Not so good & Perineum \\
& Intercostal neuralgia \\
Bad & Central Pain (after brain attack) \\
& Complete spinal cord injury \\
& Face pain \\
\hline
\end{tabular}


and to determine the degree of pain reduction (Table 2). Each patient is instructed on the method of auto-stimulation with variations in frequency and intensity. During the trial stimulation period, patients need to record the pain level objectively during and without stimulation. Testing may last up to 1 week.

\section{EFFICACY OF SCS}

\section{Post spinal surgery syndrome (PSSS)}

PSSS is defined as persistent or worsening pain of the trunk, back, neck, arms, extremities, or multiple areas after surgical correction of spinal disease. This diagnosis commonly used to describe multiple patients with varying pain patterns and represents a diverse group of patients. These patients may have varying pain generators and mechanisms of pain production including nerve injury or mechanical pain ${ }^{19)}$. Randomized control trial was grouped into repeat surgery or SCS. The results of this study were favorable for SCS. SCS proved to be superior to repeat surgery when measured by global satisfaction and analgesia $(p>0.01)$. The cross-over analysis also favored the stimulation group versus the repeat surgery group $(p=0.02)^{16)}$. This study suggests that SCS is an effective alternative to repeat surgery in patients who have failed previous lumbar spine surgery, and should be considered to be a first line treatment in this complex group of patients ${ }^{26)}$ Radicular neuropathic limb pain is a prime indication for $\mathrm{SCS}^{17}$. SCS for axial back pain has proven to be more challenging. The difficulty in relieving axial low back pain centers on the fact that the nerve fibers that must be stimulated are located in the deep lateral areas of the dorsal columns near the dorsal root entry zone and the nerve root ${ }^{6}$. Studies have shown that the earlier the patient is implanted after the failure of cervical and lumbar spine surgery, the better the chance of a good outcome ${ }^{11)}$.

\section{Complex regional pain syndrome (CRPS)}

CRPS is formally known as reflex sympathetic dystrophy, or causalgia. This problem has led to loss of function, severe pain, and tremendous expense to society. The goal of SCS in this population includes pain relief, improved blood flow, global satisfaction and increased ability to tolerate rehabilitation. The success of SCS for CRPS is well supported by high powered statistical studies $^{4}$. In a prospective, randomized trial of 36 CRPS patients, the patients that were implanted with a permanent stimulator showed long-term pain reduction and improvements in quality of life, and global satisfaction. At long-term follow up of 5 years, the group undergoing SCS and physical therapy had persistent good outcomes with pain reduction ${ }^{9}$. One common thread in all studies regarding SCS for CRPS includes the importance of moving forward with the therapy early in the course of the disease $^{9,11}$. Once the process spreads to other body parts, or the patient develops contractures, the chance of a good outcome diminishes ${ }^{18)}$.

\section{Post-herpetic neuralgia (PHN)}

Severe neuropathic pain can develop in the same area after previous herpes-zoster viral infection. This chronic and severe problem is caused by an eruption of dormant Herpes-varicella living in the dorsal root ganglia. This can lead to a chronic disruption of the nerve ending with abnormal activation of the Adelta and C fibers. Studies on the efficacy of SCS have been mixed in this condition. It does appear that the outcomes have improved over time with evolution of new technology and better programming. The factor of efficacy may be due to direct stimulation at the cord level, but it also has been theorized to be due to restoration of blood flow due to vasodilatation, changes in the sympathetic nervous system, or improved blood flow to the nerve ${ }^{10)}$. In patients who fail SCS for this condition, the method of neuromodulation may consider peripheral nerve stimulation, or intrathecal drug delivery ${ }^{7,22}$.

\section{Ischemic pain, peripheral vascular disease (PVD) and angina}

Neuropathic pain secondary to ischemia is a Food and Drug Administration (FDA) approved indication for SCS in the USA. The use of SCS for treatment of PVD has been common in Europe. The mechanism of action for the ability of SCS to relieve ischemic pain is not proven, but many have theorized it causes a change in sympathetic tone and thus increases blood flow $^{1)}$. SCS improves microcirculation, increases capillary density and increases red blood cell velocity through capillary bed ${ }^{15)}$. One of the studies indicates improved function, wound healing, and pain scores when SCS is used earlier in the treatment. Considering this information, SCS for ischemic limb pain and PVD should be considered earlier in the course of treatment. SCS has anti-ischemic effects on the myocardium and may impact survival and function in the patient with intractable angi$\mathrm{na}^{25}$. Some have theorized the mechanism to be segmental inhibition of the activity on the sympathetic nervous system to the heart, causing an increase in microcirculation, improved metabolism, and a reduction in myocardial demand of oxygen ${ }^{2)}$. SCS has shown to improve achievable cardiac work load, increase time to ischemia and angina and improve function while not blunting the patient's ability to identify significant ischemic symptoms ${ }^{20)}$.

\section{Visceral and pelvic pain}

Recent work has shown improved pain relief with SCS in those suffering from visceral abdominal pain syndromes. Pain generation may develop secondary to ischemia to the bowel, adhesions, chronic pancreatitis, or post-operative pain syndromes. Other reports have shown an improvement in symptoms using SCS with or without opioids than to opioids alone in patients with chronic pancreatitis ${ }^{3}$. Common causes of pelvic pain include interstitial cystitis, endometriosis, and post-surgical scarring. Many of these patients are treated with high dose opioids with poor results. SCS has been used successfully in these cases after 
failure of more urological or gynecological treatments. The success of treatment for pain in the interstitial cystitis patient has led to an expansion of these therapies to patients with other causes of pelvic pain ${ }^{8)}$.

\section{COMPLICATIONS OF SCS}

Like other surgical procedures, SCS can be associated with complications. The risks of the procedure must be weighed against the potential benefits. The majority of devices are placed and maintained without complications but, when they do occur, common complications are infection, dura puncture headache, increased impedance from epidural scarring, bleeding, spinal cord injury, nerve injury, lead fracture, and lead migration $^{21}$. Of these complications, the most common problem is lead migration. The vertical or lateral movement of the lead may result in loss of stimulation, and therefore causing surgical revi$\operatorname{sion}^{11}$. The incidence of these complications has varied in the reported literature, but appears to range from $1 \%$ to $23 \%$ with the most likely number of incidence being $13.5 \%$. Lead fracture appears to be the second most common complication. Inadvertent dura puncture appears to occur in less than $1 \%$ of patients ${ }^{21}$. Infection is a potential complication of SCS. Patients with high risk of infection should be optimized medically prior to implant. Bleeding is a rare but critical complication of SCS. The majority of bleeds are superficial in the wound or pocket which bring insignificant consequence. In the event of an epidural bleed, a hematoma can develop. This can lead to serious injury to the neural structures and paraplegia if not addressed immediately. Diagnosis is made by clinical suspicion and confirmed by CT. Treatment is surgical drainage ${ }^{13)}$. Spinal cord injury, nerve injury, and cord contusion are other known risks of SCS. These risks can be reduced by using a shallow needle entry, gentle lead placement, and avoidance of lead insertion by force past the area of resistance. Keeping the patient alert and conversant may also reduce the risk of injury. Internal pulse generator (IPG) are another potential cause of problem for the implanted patient. Fluid collection around IPG is a common complication. This problem, called seroma, can lead to swelling, redness and pain. Seroma formation may be reduced by minimizing tissue trauma, blunt dissection techniques, premade pocket prior to lead placement, creating an appropriately sized device pocket, and maximizing health in those with protein deficiency prior to surgery ${ }^{23)}$. Seroma may be treated by observation, pressure dressings, aspiration or by incision and drainage. Another rare but important complication is placing the generator in a depth that is not opti$\mathrm{mal}$. The generator that is placed to deep will be unable to communicate with the transdermal telemetry equipment and a generator that is too superficial may lead to erosion. The other area of concern in the immediate postoperative period is wound dehiscence. The occurrence of wound dehiscence often leads to loss of IPG and, in most cases, the entire system ${ }^{21)}$.

\section{CONTEMPORARY SCS LITERATURE}

Long-term pain relief with SCS for a number of indications has been widely documented ${ }^{11}$. A meta-analysis of the literature has shown that $50 \%$ to $60 \%$ of SCS patients report at least moderate levels of pain relief and/or have continued stimulator use at one year follow-up ${ }^{23}$. Recent data moderately supports the use of SCS for refractory pain associated with PSSS and suggests that the therapy may have a value for treating poststroke pain, central pain syndromes, and peripheral deafferentation pain ${ }^{11,13)}$. SCS has had its best success in treating nociceptive syndromes such as chronic low back pain ${ }^{13)}$; thalamic pain syndrome (probably to the frequent loss of the target cells for stimulation), postherpetic neuralgia, and pain due to spinal cord injury are not well treated with $\mathrm{DBS}^{24)}$. SCS continues to play a role in the treatment of chronic pain when other less invasive treatment modalities have been exhausted.

\section{REFERENCES}

1. Augustinsson LE, Carlsson CA, Holm J, Jivegard L : Epidural electrical stimulation in severe limb ischemia. Pain relief, increased blood flow, and a possible limb-saving effect. Ann Surg 202 : 104-110, 1985

2. Augustinsson LE, Eliasson T, Mannheimer C : Spinal cord stimulation in severe angina pectoris. Stereotact Funct Neurosurg 65 : 136-141, 1995

3. Baranidharan G, Simpson KH, Dhandapani K : Spinal cord stimulation for visceral pain--a novel approach. Neuromodulation 17 : 753-758, 2014

4. Bennett DS, Alo KM, Oakley J, Feler CA : Spinal Cord Stimulation for Complex Regional Pain Syndrome I [RSD] : a Retrospective Multicenter Experience from 1995 to 1998 of 101 Patients. Neuromodulation 2 : 202210, 1999

5. Carstens E, Gilly H, Schreiber H, Zimmermann M : Effects of midbrain stimulation and iontophoretic application of serotonin, noradrenaline, morphine and GABA on electrical thresholds of afferent C- and A-fibre terminals in cat spinal cord. Neuroscience 21 : 395-406, 1987

6. Deer T, Pope J, Hayek S, Narouze S, Patil P, Foreman R, et al. : Neurostimulation for the treatment of axial back pain : a review of mechanisms, techniques, outcomes, and future advances. Neuromodulation 17 Suppl 2: 52-68, 2014

7. Dunteman E : Peripheral nerve stimulation for unremitting ophthalmic postherpetic neuralgia. Neuromodulation 5 : 32-37, 2002

8. Hunter C, Dave N, Diwan S, Deer T : Neuromodulation of pelvic visceral pain : review of the literature and case series of potential novel targets for treatment. Pain Pract $13: 3-17,2013$

9. Kemler MA, Barendse GA, Van Den Wildenberg FA, Van Kleef M : Effect of spinal cord stimulation for chronic complex regional pain syndrome Type I : five-year final follow-up of patients in a randomized controlled trial. Journal of Neurosurgery $108: 292-298,2008$

10. Kinfe TM, Bogdan P, Vatter H : Is Spinal Cord Stimulation Useful and Safe for the Treatment of Chronic Pain of Ischemic Origin? A Review. Clin J Pain, 2015 [Epub ahead of print]

11. Kumar K, Hunter G, Demeria D : Spinal cord stimulation in treatment of chronic benign pain : challenges in treatment planning and present status, a 22-year experience. Neurosurgery $58: 481-496,2006$

12. Liu MX, Zhong J, Zhu J, Xia L, Dou NN : Treatment of postherpetic neuralgia using DREZotomy guided by spinal cord stimulation. Stereotact Funct Neurosurg 93 : 178-181, 2015

13. Mekhail NA, Mathews M, Nageeb F, Guirguis M, Mekhail MN, Cheng J : Retrospective review of 707 cases of spinal cord stimulation : indications and complications. Pain Pract 11 : 148-153, 2011

14. Melzack R, Wall PD : Pain mechanisms : a new theory. Science 150 : $971-$ 979, 1965 
15. Naoum JJ, Arbid EJ : Spinal cord stimulation for chronic limb ischemia. Methodist Debakey Cardiovasc J 9 : 99-102, 2013

16. North RB, Kidd DH, Farrokhi F, Piantadosi SA : Spinal cord stimulation versus repeated lumbosacral spine surgery for chronic pain : a randomized, controlled trial. Neurosurgery 56 : 98-106, 2005

17. North RB, Kidd DH, Olin J, Sieracki JM, Farrokhi F, Petrucci L, et al. : Spinal cord stimulation for axial low back pain : a prospective, controlled trial comparing dual with single percutaneous electrodes. Spine 30 : 14121418,2005

18. Prager JP : What does the mechanism of spinal cord stimulation tell us about complex regional pain syndrome? Pain Med 11 : 1278-1283, 2010

19. Rigoard P, Jacques L, Delmotte A, Poon K, Munson R, Monlezun O, et al. : An algorithmic programming approach for back pain symptoms in failed back surgery syndrome using spinal cord stimulation with a multicolumn surgically implanted epidural lead : a multicenter international prospective study. Pain Pract 15 : 195-207, 2015

20. Saraste A, Ukkonen H, Varis A, Vasankari T, Tunturi S, Taittonen M, et al. : Effect of spinal cord stimulation on myocardial perfusion reserve in patients with refractory angina pectoris. Eur Heart J Cardiovasc Imaging 16 : 449-455, 2015
21. Shamji MF, Westwick HJ, Heary RF : Complications related to the use of spinal cord stimulation for managing persistent postoperative neuropathic pain after lumbar spinal surgery. Neurosurg Focus 39 : E15, 2015

22. Shimoji K: Effect of anesthetics on central and peripheral nervous system. Masui 21 : 1293-1298, 1972

23. Slavin KV, Vaisman J, Pollack KL, Simopoulos TT, Kowlowitz E, Weinand ME, et al. : Treatment of chronic, intractable pain with a conventional implantable pulse generator : a meta-analysis of 4 clinical studies. Clin J Pain 29 : 78-85, 2013

24. Smits H, van Kleef M, Holsheimer J, Joosten EA : Experimental spinal cord stimulation and neuropathic pain : mechanism of action, technical aspects, and effectiveness. Pain Pract 13 : 154-168, 2013

25. Tsigaridas N, Naka K, Tsapogas P, Pelechas E, Damigos D : Spinal cord stimulation in refractory angina. A systematic review of randomized controlled trials. Acta Cardiol 70 : 233-243, 2015

26. Van Buyten JP, Al-Kaisy A, Smet I, Palmisani S, Smith T: High-frequency spinal cord stimulation for the treatment of chronic back pain patients : results of a prospective multicenter European clinical study. Neuromodulation 16 : 59-65, 2013 